\title{
Strain Relaxation Mechanisms in Coherent and Incoherent Ge(Si)/Si Islands
}

\author{
J. Zou, ${ }^{* * * * *}$ X. Z. Liao, ${ }^{* *}$ D. J. H. Cockayne, ${ }^{* * *}$ and Z. M. Jiang**** \\ * The University of Queensland, Division of Materials and Centre for Microscopy and \\ microanalysis, Brisbane, QLD 4072, Australia \\ ** Los Alamos National Laboratory, Division of Materials Science and Technology, Los \\ Alamos, NM 87545 \\ *** $\quad$ Oxford University, Department of Materials, Oxford, OX1 3PH, England \\ **** Fudan University, Department of Physics, Shanghai 200433, China
}

Strain relaxation in semiconductor heterostructures has received enormous attention for decades due to its scientific and technological importance. This is particularly true for high strain (greater than $2 \%$ ) heterostructures, since islands can be produced by a self-assembly process in this strain range which opens up possibilities for the production of a wide range of new opto/electronic devices. The well accepted model of strain relaxation in these systems is that islands are produced by the Stranski-Krastanow mode of self-assembly growth, in which the growth starts with the layer-bylayer growth followed by island growth when the accumulated misfit strain becomes sufficient high. With further growth of the islands, misfit dislocations are generated at the island edge to relieve misfit strain since the island edge possesses the highest strain. In this study, we demonstrate two alternative strain relaxation mechanisms at the different stages of the island growth.

Ge was grown on $\mathrm{Si}(001)$ by means of solid-source molecular beam epitaxy at $700^{\circ} \mathrm{C}$ with a nominal growth thickness of $1.4 \mathrm{~nm}$ and a growth rate of $0.02 \mathrm{~nm} / \mathrm{min}$. TEM specimens were prepared by mechanical thinning followed by precision ion polishing. These specimens were investigated in a range of transmission electron microscopes.

Extensive TEM investigation indicates that in this system (i) the base diameter of coherent islands can be as large as $\sim 100 \mathrm{~nm}$ and (ii) trenches are formed around islands. Detailed study indicates that trenches have similar dimensions regardless of the size of the islands. Figure 1 shows examples of (a) a typical coherent island and (b) a typical incoherent island where many misfit dislocations are seen near the island/substrate interface. It is reasonable to assume that the Si originally in the trench has diffused into the island. To prove this, energy filtered imaging (EFI) techniques were employed using a Gatan EFI system. Figure 2 is (a) a Si map formed with electrons with an energy-loss $99 \mathrm{eV}$ and (b) a Ge map created with electrons with an energy-loss $1217 \mathrm{eV}$, showing Si within the island although pure Ge was deposited on the Si substrate. It is clear that Si moves into the islands causing alloying which reduces the strain energy of the islands. This observation is in agreement with the model in which strain relaxation during growth of coherent islands is achieved through alloying. This strain relief mechanism is likely to be enhanced for high temperature growth.

With further growth of the islands, coherent islands collapse by the introduction of misfit dislocations. It is generally believed that these misfit dislocations originate from the edges of islands where the strain is the highest. If this were the only mechanism for generation of misfit dislocations, one would expect to see distances of more than $\sim 100 \mathrm{~nm}$ between the two innermost misfit dislocations. However, the experimental result shown in Fig. 2(b) is in conflict with this suggestion, unless it is assumed that misfit dislocations may climb in the interface (which is an energetically unfavorable process). In many islands, we have found stacking faults with one end terminating at the island surface and the other end associated with a partial dislocation within the island (in most cases, terminating near the interface). Figure 3 shows a case in which all misfit dislocations are partial dislocations with stacking faults. It is noted that the distance between the two innermost dislocations is $\sim 50 \mathrm{~nm}$ which is well below $\sim 100 \mathrm{~nm}$ (the critical base diameter for the collapse of coherent islands). This result suggests that misfit dislocations might be generated at the island surface and glide to the interface. To explore this possibility, extensive HREM investigations were 
performed to determine the character of these partial dislocations. Figure 4(a) shows an island that has two partial dislocations, with one near the interface and the other near the island surface. Figures 4(b) and (c) are enlarged HREM images that show the core structure of these partials. In Fig. 4(b), the core of the partial (near the interface) is clearly seen, and a Burgers circuit shows it to be $30^{\circ}$ in character. The partial that is near the island surface also has a $30^{\circ}$ character, but its blurred image is consistent with the dislocation line being curved, as for a half loop. Based on the facts that (i) for the compressive strained layer grown on a [001] substrate, a $30^{\circ}$ partial leads the movement of a dissociated $60^{\circ}$ dislocation; and (ii) $30^{\circ}$ partial loops are seen near the island surface, we propose that partial misfit dislocations can originate from the island surface, and move down to the interface to relieve the strain which is concentrated there. Possible mechanisms for overcoming the energy barrier which exists in this model are local fluctuations in the strain caused by topological and compositional changes during growth.

In conclusion, it is evident that strain relaxation in semiconductor islands can be achieved both through alloying during the coherent island growth and by the introduction of misfit dislocations from the island surface.

The Australian Research Council is thanked for providing financial support. This work is also supported by the National Nature Science Foundation of China (Grant No 10174012).
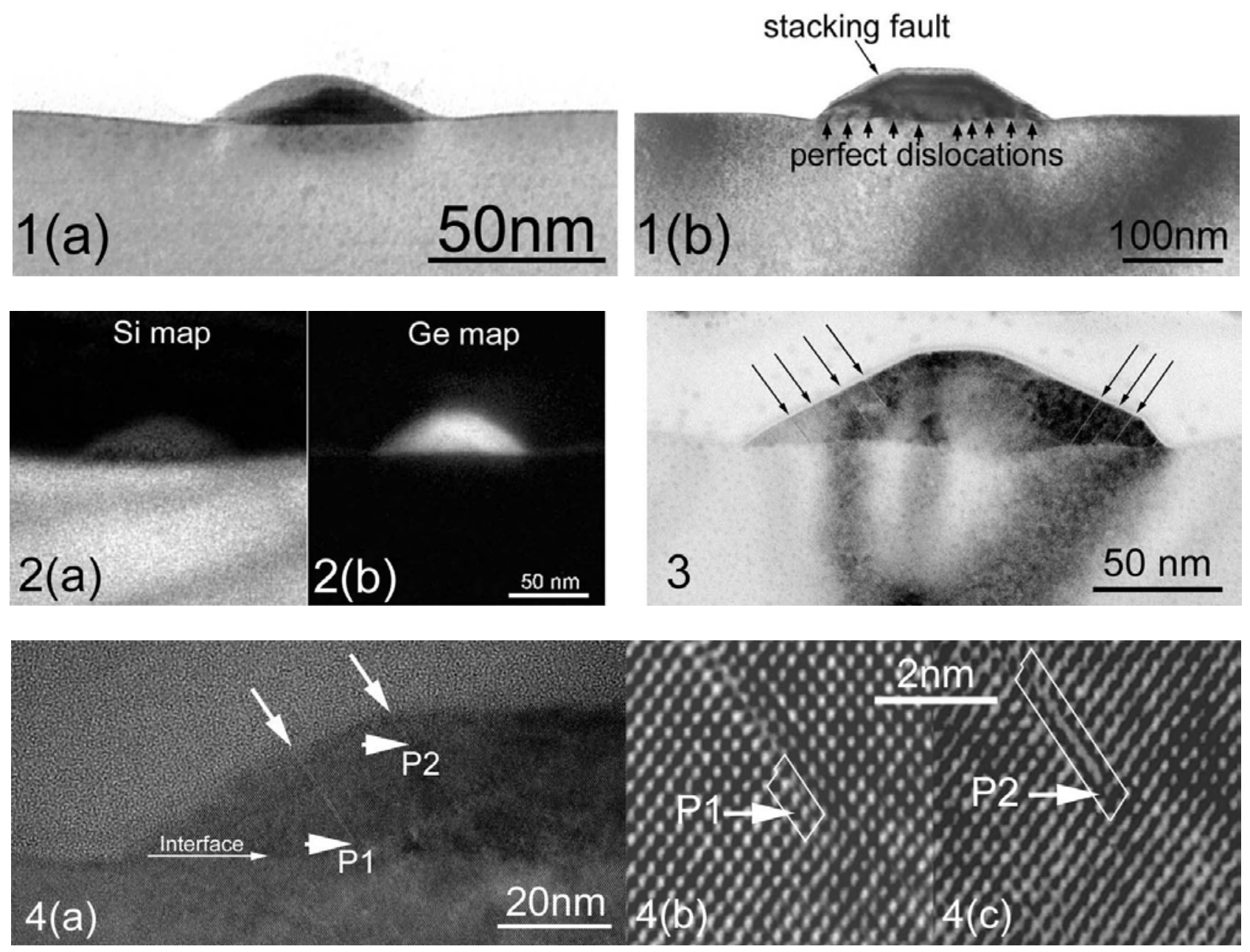